\title{
L'analyse des débits des rivières issues du plateau de Millevaches lors de la crue du 4 octobre 1960
}

\author{
Flow rate analysis for rivers issuing from \\ the Millevaches plateau \\ during the 4th October 1960 flood
}

PAR
P. PELLECUER
ET
F. BARRIËRE

INGÉNIEURS A LA $4^{\circ}$ CIRCONSCRIPTION ÉLECTRIQUE, LIMOGES

\begin{abstract}
- Etendue géographique du phénomène et points de mesure pris en compte.

- Eléments de base pour l'évaluation des hauteurs et des débits: enregistrements, courbes expérimentales $Q=\mathrm{f}(\mathrm{h})$, formules.

- Résultats d'ensemble: hydrogrammes de hauteurs et de débits, débits maximaux atteints, comparaison des volumes roulés et des volumes précipités dans certains temps-types.

- Etude plus particulière du phénomène dans le bassin de la Corrèze. Influence des facteur's topographiques et géologiques dans les pointes de débit.

- Conclusion : comment se situe le phénomène par rapport aux grandes crues connues; essai d'évaluation d'une durée de retour; leçons pratiques à tirer pour l'exploitation des stations de jaugeage.
\end{abstract}

\begin{abstract}
Geographical extent of the phenomenon and measurements considered.

Basic data for stage and discharge evaluation: recordings, experimental $Q=f(h)$ curves, formulae.

Overall results: Stage/discharge hydrographs, maximum flow rates, comparison between "rolled" and precipitated volumes for varions standard times.

Individual study of the phenomenon in the Corrèze basin. Influence of topographical and geological factors on flow peaks.

Conclusion: Relationship between the phenomenon and the major known floods; attempt to forecast its recurrence; practical information derived for the operation of gauging stations.
\end{abstract}

\section{1. - L'ETENDUE GÉOGRAPHIQUE DU PHENOMÈNE (voir fig. 1)}

Le phénomène que nous étudions est limité géographiquement à une bande relativement étroite, orientée sud-ouest nord-est, dont le grand axe, de $150 \mathrm{~km}$ environ, est constitué par la ligne Brive-Teillet, et dont la largeur est d'environ $45 \mathrm{~km}$ (distance d'Eymoutiers à Ussel). 


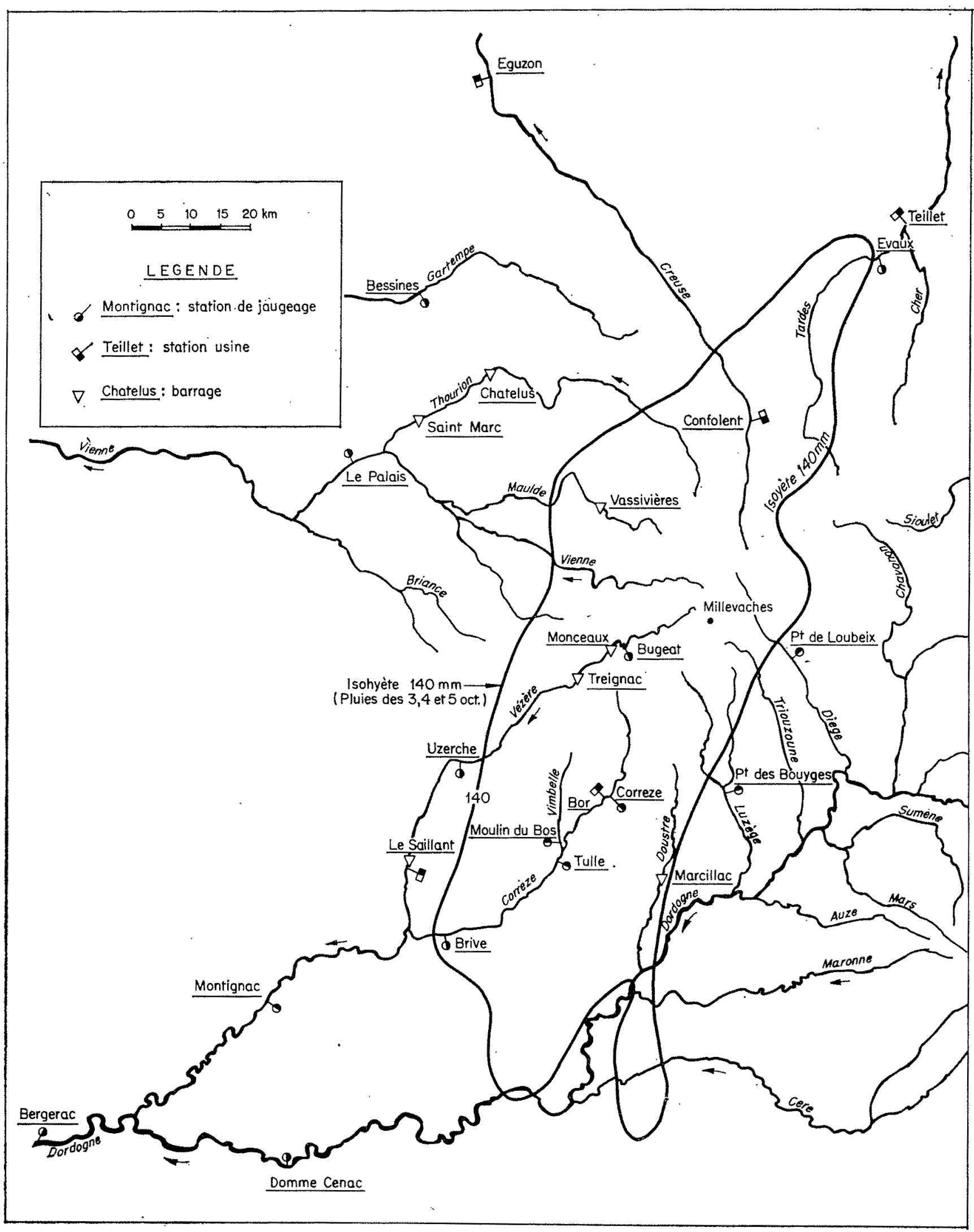

Frg. 1

Carte de situation des points de mesure. 


\section{2. - BASSINS INTÉRESSÉS ET POINTS DE MESURE (voir fig. 1)}

2.1 L'isohyète de $140 \mathrm{~mm}$ (pluie des 3,4 et 5 octobre) délimite les bassins les plus touchés. En opérant une rotation dans le sens des aiguilles d'une montre autour du bourg de Millevaches, avec comme rayon d'origine Millevaches-Tulle, nous rencontrons :

\begin{tabular}{|c|c|c|}
\hline Rivière & En amont de & $\begin{array}{c}\text { Pour } \\
\text { (en } \mathrm{km}^{2} \text { ) }\end{array}$ \\
\hline La Corrèze.... & Brive $\ldots \ldots \ldots$ & 1000 \\
\hline La Vézère..... & Uzerche. . . . . . . & 600 \\
\hline La Vienne.... & Eymoutiers. . . . . & 200 \\
\hline La Maulde.... & Peyrat. . . . . . . . . & 200 \\
\hline Le Taurion.... & Chatain. . ........ & 80 \\
\hline La Creuse.... & Ahun. . . . . . & 500 \\
\hline $\begin{array}{l}\text { La Tardes, et } \\
\text { La Vouèze.... }\end{array}$ & Evaux. . . . & 900 \\
\hline La Diège...... & Pont de Loubeix.. & 220 \\
\hline La Luzège.... & Pont des Bouyges. & 250 \\
\hline \multirow[t]{2}{*}{ Le Doustre... } & \multirow[t]{2}{*}{ Marcillac. . . . . . . } & 150 \\
\hline & & 4100 \\
\hline
\end{tabular}

Au-dessous de $120 \mathrm{~mm}$ de précipitation et de $250 \mathrm{l} / \mathrm{s} . \mathrm{km}^{2}$ de débit spécifique, on ne peut plus parler de phénomène exceptionnel; c'est pourquoi nous limitons à cette isohyète et à environ $5000 \mathrm{~km}^{2}$ la superficie intéressée.

\subsection{Les points de mesure (voir fig. 2)}

Nous avons étudié le passage de la crue en seize points. Par points, il faut entendre soit des stations de jaugeage, soit des aménagements hydroélectriques (évacuateurs de crue des barrages et groupes).

Nous distinguerons, du point de vue des effets dynamiques de la crue, les groupes de bassins ci-après :

- En premier lieu, l'ensemble du bassin de la Corrèze, qui a certainement présenté sur ses $1000 \mathrm{~km}^{2}$ les caractères les plus homogènes en même temps que les intensités spécifiques les plus élevées. Il était d'autant plus intéressant à examiner qu'il est un des très rares du Massif Cientral encore vierge de tout aménagement important. Nous l'étudions en quatre points :

Corrèze, sur la Corrèze (station de jaugeage et barrage)........ $167 \mathrm{~km}^{2}$ Moulin du Bos, sur la Vimbelle, principal affluent rive droite de la Corrèze avant Tulle......... $140 \mathrm{~km}^{2}$ Tulle, sur la Corrèze......... $370 \mathrm{~km}^{2}$ Brive, sur la Corrèze......... $947 \mathrm{~km}^{2}$

- Ensuite, sur le bassin de la Vézère, où les intensités des débits de pointe ont été moindres que sur la Corrèze et où la crue a été très fortement écrêtée par les retenues de Montceaux et Treignac, nous étudions la crue en quatre points également :

Bugeat sur la Vézère......... $143 \mathrm{~km}^{2}$

Uzerche, sur la Vézère........ $601 \mathrm{~km}^{2}$

Le Saillant, sur la Vézère, à l'usine

d'E.D.F.. . . . . . . . . . . $950 \mathrm{~km}^{2}$

Montignac, sur la Vézère, à l'aval

du confluent de la Corrèze... $3126 \mathrm{~km}^{2}$

- Sur la Creuse, qui a atteint des chifrres relativement considérables, nous étudions la crue en deux points :

Confolent, à l'usine d'E.D.F.... $200 \mathrm{~km}^{2}$

Eguzon, à l'usine d'E.D.F..... $2400 \mathrm{~km}^{2}$

- Sur le bassin du Cher, c'est à la station d'Evaux $\left(860 \mathrm{~km}^{2}\right)$ que nous étudions le phénomène très spectaculaire de la Tardes (après le confluent Vouèze), laquelle détient le record relatif de cette crue avec un débit de pointe soixante-huit fois supérieur à son module. Pour le Cher proprement dit, le point choisi est l'usine E.D.F. de Teillet $\left(1600 \mathrm{~km}^{2}\right)$ où le débit de pointe a été constitué pour les trois-quarts par l'apport de la Tardes, alors que le haut Cher avait une crue insignifiante.

- Enfin pour le passage de la crue dans les affluents rive droite de la Dordogne, issus du versant sud et sud-ouest du plateau de Millevaches, nous choisissons les deux points de:

Pont de Loubeix, sur la Diège... . $225 \mathrm{~km}^{2}$ Pont de Bouyges, sur la Luzège. . $252 \mathrm{~km}^{2}$ 


\begin{tabular}{|c|c|c|c|c|c|c|c|c|c|c|c|c|c|c|c|c|c|}
\hline \multirow{3}{*}{ Rivière } & \multirow{3}{*}{$\begin{array}{l}\text { Station } \\
\text { ou usine }\end{array}$} & \multirow{3}{*}{$\begin{array}{c}\text { BASSIN } \\
\text { versANT } \\
\left({\left.\operatorname{en~} \mathrm{km}^{2}\right)}^{2}\right.\end{array}$} & \multirow{3}{*}{$\begin{array}{l}\text { DÉBIT } \\
\text { DE BASE } \\
\left(\mathrm{m}^{\mathrm{3}} / \mathrm{s}\right)\end{array}$} & \multicolumn{3}{|c|}{ DÉBITS } & \multirow{3}{*}{$\frac{Q_{\max }}{Q_{J}}$} & \multirow{3}{*}{$\begin{array}{c}\text { MODULE } \\
1 / \mathrm{s} \cdot \mathrm{km}^{2}\end{array}$} & \multirow{3}{*}{$\frac{Q_{\max }}{\text { Module }}$} & \multirow{2}{*}{\multicolumn{3}{|c|}{ LAMES D'EAU }} & \multirow{3}{*}{$\left|\begin{array}{c}\text { Hautrü } \\
\text { max. } \\
a \\
l^{1} \text { ćehelle } \\
\text { (en m) }\end{array}\right|$} & \multirow{2}{*}{\multicolumn{3}{|c|}{$\begin{array}{c}\text { PLUS FORTE CRUE CONNUE } \\
(d)\end{array}$}} & \multirow{3}{*}{$\begin{array}{l}\text { Observations } \\
\text { relatives aux stations }\end{array}$} \\
\hline & & & & \multicolumn{2}{|c|}{ 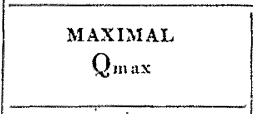 } & \multirow{2}{*}{$\mid \begin{array}{c}\text { JOUR- } \\
\text { NALIER } \\
Q_{J} \\
\left(\mathrm{~m}^{3} / \mathrm{s}\right) \\
(a) \\
\end{array}$} & & & & & & & & & & & \\
\hline & & & & $\left(\mathrm{m}^{3} / \mathrm{s}\right)$ & $1 / \mathrm{s} . \mathrm{km}^{2}$ & & & & & $\begin{array}{l}\mathrm{L}_{e} \\
(b)\end{array}$ & $\begin{array}{l}\mathrm{L}_{p} \\
(c)\end{array}$ & $\mathrm{K}_{72}=\frac{\mathrm{L}_{\mathrm{o}}}{\mathrm{L}_{\mathrm{p}}}$ & & \begin{tabular}{c|c}
$\mathrm{H}$ \\
$(\mathrm{en} \mathrm{m})$
\end{tabular} & $\begin{array}{l}Q_{\max } \\
\left(\mathrm{m}^{3} / \mathrm{s}\right) \\
\end{array}$ & \begin{tabular}{|c|} 
Date \\
et période
\end{tabular} & \\
\hline $\begin{array}{c}1 \\
\text { Corrèze. }\end{array}$ & $\begin{array}{c}2 \\
\text { Corrèze. . . . . }\end{array}$ & $\begin{array}{c}3 \\
167\end{array}$ & $\begin{array}{l}4 \\
4,5\end{array}$ & $\begin{array}{c}5 \\
189\end{array}$ & $\begin{array}{c}6 \\
1130\end{array}$ & $\begin{array}{l}7 \\
121\end{array}$ & $\begin{array}{c}8 \\
1,54\end{array}$ & $\begin{array}{r}9 \\
33\end{array}$ & $\begin{array}{l}10 \\
34\end{array}$ & $\begin{array}{r}11 \\
107\end{array}$ & \begin{tabular}{c|}
12 \\
196
\end{tabular} & $\begin{array}{c}13 \\
0,55\end{array}$ & $\begin{array}{r}14 \\
4,15\end{array}$ & $\begin{array}{r}15 \\
3,50\end{array}$ & $\begin{array}{r}16 \\
105\end{array}$ & \begin{tabular}{|c|}
17 \\
$8-12-44$ \\
$(40$ ans $)$
\end{tabular} & \begin{tabular}{l}
\multicolumn{1}{c}{18} \\
Au barrage de Corrèze \\
Débit max. $186 \mathrm{~m}^{3} / \mathrm{s}$ \\
$1060 \mathrm{l} / \mathrm{s} . \mathrm{km}^{2}$ \\
B.V. 175.
\end{tabular} \\
\hline Vimbelle. . & Moulin du Bos.... & 140 & 2,3 & 183 & 1300 & 103 & 1,77 & 25 & 52 & 95 & 182 & 0,52 & 3,90 & & & & Station créće en 1958. \\
\hline Corrèze. . & Tulle...... & 371) & 7 & 450 & 1220 & 293 & 1,54 & 26,5 & 46 & 103 & 188 & 0,55 & 5,43 & 3,75 & 265 & $\begin{array}{l}8-12-44 \\
(40 \text { ans })\end{array}$ & \\
\hline Corrèze. . & Brive. & 947 & 17 & 750 & 790 & 610 & 1,23 & 23 & 34 & 90 & 168 & 0,54 & $5,15^{(1)}$ & $\left|3,56^{(1)}\right|$ & 460 & \begin{tabular}{|c|}
$18-9-21$ \\
$(40$ ans $)$
\end{tabular} & $\begin{array}{l}\text { (1) Echelle du Service d'An- } \\
\text { nonce des Crues. }\end{array}$ \\
\hline Vézère. . . & Bugeat. . & 143 & 2 & 115 & 805 & 90 & 1,28 & 33 & 24 & 113 & 200 & 0,56 & 2,52 & 2,02 & 67 & $\begin{array}{l}8-12-44 \\
(25 \text { ans })\end{array}$ & \\
\hline Vézère. . . & Uzerche $(e)$. . & 601 & 15 & 410 & 680 & 295 & 1,39 & 25 & 27 & 80 & 175 & 0,45 & $3,85(2)$ & $2,85(2)$ & 275 & $\begin{array}{c}8-12-44 \\
(40 \text { ans }) \\
\end{array}$ & $\begin{array}{l}\text { (2) Echelle du Service d’An- } \\
\text { nonce des Crues. }\end{array}$ \\
\hline Vézère. . . & Le Saillant $(e)$. & 950 & 20 & 432 & 455 & 366 & $\overline{1,18}$ & 23 & 20 & 64 & $\overline{158}$ & 0,41 & & & & & \\
\hline Vézère. . . & Montignac $(e)$. & 3126 & 45 & 1220 & 390 & 1020 & 1,18 & 18 & 22 & 63 & 137 & 0,46 & $8,90^{(3)}$ & $7,40^{(3)}$ & 900 & $\begin{array}{c}9-12-44 \\
(63 \text { ans })\end{array}$ & $\begin{array}{l}\text { (3) Echelle aval du pont. } \\
\text { Cotes à l'échelle amont (A. C.) } \\
1944-7,90 ; 1960-9,43 .\end{array}$ \\
\hline Luzège. . . & Pont de Bouyges. & 252 & 3 & 210 & 830 & 149 & 1,41 & 24,5 & 34 & 84 & 125 & 0,67 & 2,90 & & & $\begin{array}{l}8-12-44(4) \\
(40 \text { ans })\end{array}$ & $\begin{array}{l}\text { (4) A la station de Lapleau } \\
\left(402 \mathrm{~km}^{2}\right) \text {. }\end{array}$ \\
\hline Diège. . & Pont de Loubeix. & 225 & & 165 & 730 & 75 & 2,20 & 20 & 36 & & & & 3,00 & & & & $\begin{array}{l}\text { Ce module de } 20 \text { est un ordre } \\
\text { de grandeur. Les autres chif- } \\
\text { fres seront extraits du rap- } \\
\text { port de M. d'Harcourt. }\end{array}$ \\
\hline Tardes. . & Evaux. . . . . . . . & 860 & 6,0 & 650 & 760 & 455 & 1,67 & 9,5 & 68 & 66 & 132 & 0,50 & 4,94 & & & & \\
\hline Cher. .... & Teillet. & 1600 & 10 & 845 & 528 & 700 & 1,21 & 9 & 58 & 65 & 105 & 0,63 & & & 680 & $\begin{array}{c}4-5-40 \\
(40 \text { ans }) \\
\end{array}$ & \\
\hline Creuse. .. & Confolent. . . . . : & 200 & & 164 & 820 & 95 & $\overline{1,72}$ & 19,5 & 42 & 80 & & & & & & & \\
\hline Creuse. . . & Eguzon. & 2400 & 21 & 1150 & 479 & 715 & 1,61 & 12 & 40 & 50 & & & & & 620 & $\begin{array}{l}18-12-52 \\
(35 \text { ans })\end{array}$ & \\
\hline Gartempe. & Bessines. . . . . . . & 575 & 5 & 79 & 137 & 58 & $\overline{1.36}$ & 13 & 10 & 17 & & & & & & & \\
\hline$\overline{\text { Vienne. .. }}$ & Le Palais $(e) \ldots \ldots$ & 2300 & 54 & 600 & 263 & 446 & $\overline{1,35}$ & 18 & 15 & 27 & & & & & 600 & $\begin{array}{l}8-12-44 \\
(35 \text { ans })\end{array}$ & \\
\hline $\begin{array}{l}\text { mum d } \\
\text { moyen } \\
\text { base. }\end{array}$ & $\begin{array}{l}\text { (a) Il s'agit du débi } \\
\text { le la crue. } \\
\text { (b) Pour le calcul d } \\
\text { des } 72 \text { heures qui s } \\
\text { (c) } \mathrm{L}_{p}=\text { total en } \mathrm{mn}\end{array}$ & $\begin{array}{l}\text { it moyen } \\
\text { le } \mathrm{L}_{e} \text { (lan } \\
\text { suivent } \mathrm{l} \\
\mathrm{m} \text { de la } \mathrm{p}\end{array}$ & $\begin{array}{l}\text { en } r \\
\text { me d'e } \\
\text { e dém } \\
\text { pluie } t\end{array}$ & $\begin{array}{l}\text { /s du jo } \\
\text { écoulée } \\
\text { rage de } \\
\text { abée sur }\end{array}$ & $\begin{array}{l}\text { our où s } \\
\text { e) il est } \\
\text { la crue, } \\
\text { le bassir }\end{array}$ & $\begin{array}{l}\text { s'est pr } \\
\text { tenu c } \\
\text {, ampu }\end{array}$ & $\begin{array}{l}\text { coduit } \\
\text { ompte } \\
\text { até du }\end{array}$ & $\begin{array}{l}\text { le maxi- } \\
\text { du débit } \\
\text { débit de } \\
5 \text { octobre. }\end{array}$ & & de $p$ & $\begin{array}{l}\text { Il s' } \\
\text { it de } \\
\text { Ces } \\
\text { e par } \\
\text { à l'a }\end{array}$ & $\begin{array}{l}\text { agit de } 1 \\
\text { epuis la } \\
\text { stations } \\
\text { rtie du } \\
\text { aval. }\end{array}$ & $\begin{array}{l}\text { la plus } \mathrm{fc} \\
\text { création } \\
\text { ou usin } \\
\text { volume }\end{array}$ & $\begin{array}{l}\text { forte cru } \\
\text { n des se } \\
\text { nes étaie } \\
\text { de la cr }\end{array}$ & $\begin{array}{l}\text { e antér } \\
\text { ervices } \\
\text { ent infl } \\
\text { ue et }\end{array}$ & $\begin{array}{l}\text { érieurement } \\
\text { s d'ètude } \\
\text { fluencées p } \\
\text { ont contril }\end{array}$ & $\begin{array}{l}\text { bien connue en hauteur } \\
\text { les débits. } \\
\text { ar des retenues qui ont } \\
\text { bué à affaiblir les débits }\end{array}$ \\
\hline
\end{tabular}


Nous ajoutons à cette liste deux points choisis volontairement en dehors du phénomène :

Le Palais, sur la Vienne $\left(2200 \mathrm{~km}^{2}\right)$, où la crue fut considérablement écrêtée et modifiée par un important stockage dans la retenue de Vassi- vières et dans celles de la Maulde et du Taurion.

Bessines, sur la Gartempe $\left(575 \mathrm{~km}^{2}\right)$, qui permet de constater qu'en dehors de la bande de territoires intéressée par le phénomène, les débits n'ont pas dépassé la valeur d'une crue annuelle.

\section{3. - ELEMENTS DE BASE POUR L'ÉVALUATION DES HAUTEURS ET DES DÉBITS}

Dans chacun des points retenus, on a déterminé les hauteurs et débits horaires, au passage de la crue, et l'évaluation de chaque débit maximal au droit des stations de jaugeage a fait l'objet d'une note de calcul.

Pour les hauteurs il n'y a en général jamais de difficulté à préciser la hauteur maximale atteinte, même en l'absence d'enregistrement. Les appareils de notre service (mis à part celui de Brive qui fut emporté par la crue), même submergés par celle-ci, ont fourni au moins trois données essentielles: le départ de la crue, la vitesse de montée dans la première partie de celle-ci et, au bout d'un temps variant entre 12 et 48 heures, l'allure de la décrue après remise en service. De ce fait la reconstitution de l'hydrogramme des hauteurs n'a pas posé de difficultés insurmontables.

Pour l'évaluation des débits, les courbes de tarage $Q=f(h)$ des stations ont dû être évidemment extrapolées pour les hauteurs maximales atteintes. Nous l'avons fait par les méthodes classiques (sections mouillées et extrapolation de la courbe des vitesses moyennes). Nous avons utilisé en outre, dans la majorité des cas, en dehors de la section normale au droit de l'échelle principale, une section auxiliaire où les mêmes calculs ont été conduits de manière à obtenir un recoupement de l'évaluation du débit maximal instantané.

\section{4. - SITUATION HYDROLOGIQUE DES BASSINS AVANT LA CRUE}

L'hydraulicité des mois précédant la crue préparait un terrain favorable au ruissellement de celle-ci. En effet, après un mois de décembre 1959 durant lequel les rivières ont écoulé un débit double de la moyenne, le premier trimestre 1960 donna une hydraulicité légèrement excédentaire.

Pour les quatre mois suivants par contre (printemps et début de l'été), les coefficients d'hydraulicité tombent au-dessous de la moyenne, mais sans que l'on puisse parler de sécheresse, d'autant qu'un automne particulièrement pluvieux devait suivre avec des hydraulicités des mois d'août et septembre atteignant et même dépassant $200 \%$. Signalons en passant l'hydraulicité d'octobre 1960 (700 à $1000 \%$ selon les stations), sans précédent depuis le début d'exploitation des stations, hydraulicité dont la responsabilité incombe non seulement à la crue du 4 octobre, mais aussi aux pluies quasi quotidiennes de ce mois qui ont donně pour les hauts bassins de la Vézère et de la Corrèze le total mensuel de $430 \mathrm{~mm}$.

En fait, on peut dire que la préparation d'un terrain favorable à un ruissellement supérieur à celui de la saison a commencé d'une manière active avec les orages de fin août. La moitié des précipitations du mois d'août sont tombées à partir du 26 août. Du 26 août au 30 septembre, l'hydraulicité relative des débits à Brive a été de $263 \%$. Ces orages ont préparé un tremplin dont l'humidité a été entretenue par les pluies presque ininterrompues jusqu'à la veille de la crue. Il a plu en effet durant vingt-cinq jours, du 25 août au 30 septembre.

Le débit de base le 3 octobre au matin est reproduit pour chaque station dans la colonne 4 du tableau ci-annexé (voir fig. 2). Il est de l'ordre du D.C. 6 d'une courhe globale des débits classés. 


\section{5. - LES HYDROGRAMMES DE HAUTEURS}

Les hydrogrammes de la crue pour la Vézère, à Bugcat et à Montignac, la Corrèze à Corrèze et la Tardes à Evaux sont reproduits sur les figures 3, $4,5,6$.
Quelques données sur les amplitudes atteintes et les vitesses de montée de l'eau sont rassemblées dans le tableau ci-dessous :

\begin{tabular}{|c|c|c|c|c|c|c|c|c|}
\hline \multirow{2}{*}{ Rivière } & \multirow{2}{*}{ Station } & \multirow{2}{*}{$\begin{array}{c}\text { Bassin } \\
\text { versant } \\
\text { (en } \mathrm{km}^{2} \text { ) }\end{array}$} & \multirow{2}{*}{$\begin{array}{c}\text { Cote } \\
\text { initiale } \\
\text { (en m) }\end{array}$} & \multirow{2}{*}{$\begin{array}{c}\text { Cote } \\
\text { du } \\
\text { maximum } \\
(\mathrm{en} \mathrm{m})\end{array}$} & \multirow{2}{*}{$\begin{array}{l}\text { Amplitude } \\
\text { (en m) }\end{array}$} & \multirow{2}{*}{$\begin{array}{c}\text { Temps } \\
\text { de montée } \\
\text { (en heures) }\end{array}$} & \multicolumn{2}{|c|}{ Vitesse de montée } \\
\hline & & & & & & & $\begin{array}{c}\text { moyenne } \\
(\mathrm{en} \mathrm{cm} / \mathrm{h})\end{array}$ & $\begin{array}{l}\operatorname{maximale} \\
(\mathrm{en} \mathrm{cm} / \mathrm{h})\end{array}$ \\
\hline Corrèze. . . . & Corrèze. . . . . . . & 167 & 0,66 & 4,15 & 3,49 & 20 & 18 & 36 \\
\hline Vimbelle. . . & Moulin du Bos... & 140 & 0,36 & 3,90 & 3,54 & 18 & 20 & 50 \\
\hline Corrèze. . . & Tulle. . . . . . . & 370 & 0,45 & 5,43 & 4.98 & 20 & 25 & 50 \\
\hline Vézère. . . & Bugeat. . . ..... & 143 & 0,54 & 2,51 & 1,97 & 18 & 11 & 16 \\
\hline Vézère. . . & Montignac. . . . & 3126 & 0,84 & 8,90 & 8,06 & 34 & 24 & 40 \\
\hline Luzège. . . . & Pont des Bouyges & 252 & 0,60 & 2,90 & 2,30 & 24 & 10 & 20 \\
\hline Tardes. . . . & Evaux. ......... & 860 & 0,65 & 4,95 & 4,30 & 15 & 29 & 50 \\
\hline Corrèze. . . . & Brive. . . . . . . . & 947 & 0,60 & 5,20 & 4,60 & 13 & 35 & 68 \\
\hline
\end{tabular}

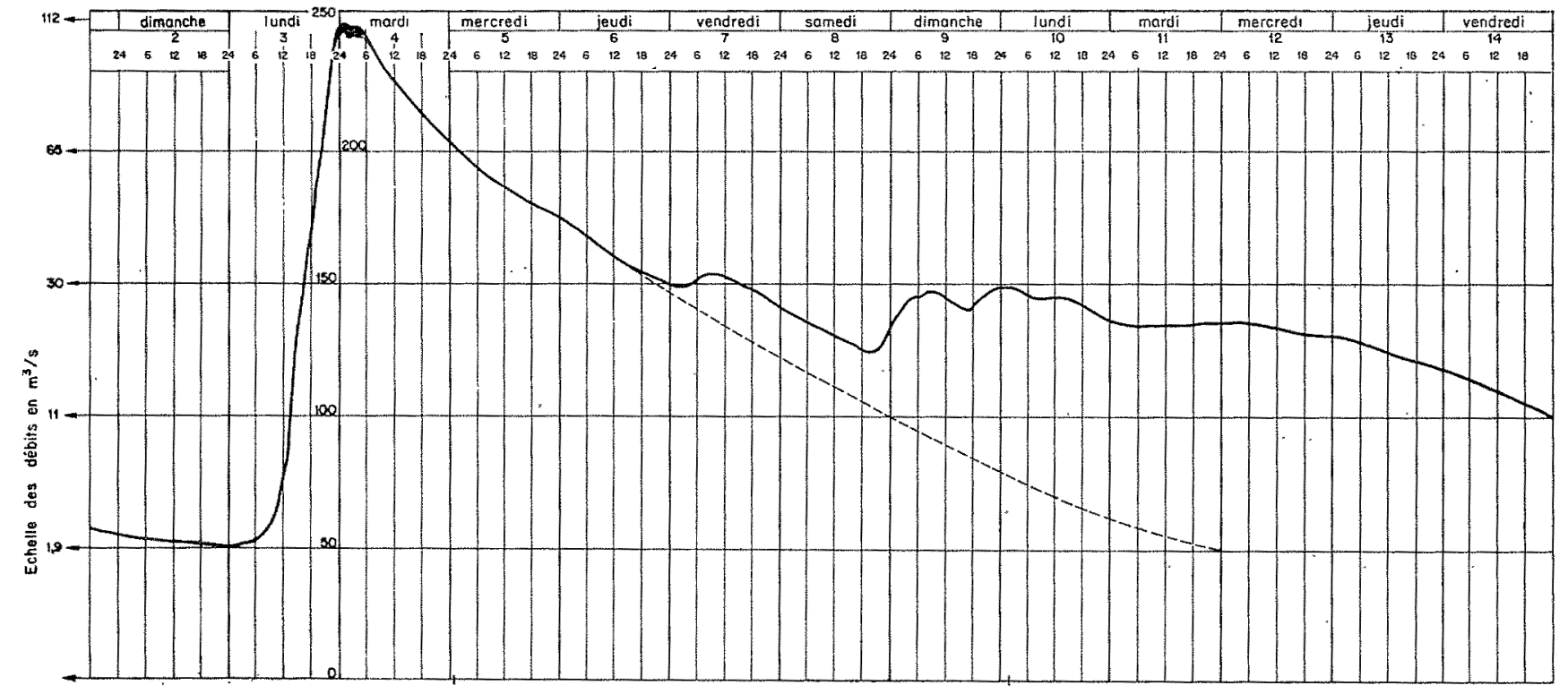

Fig. 3

Crue du 4-10-1960

La Vézère à Bugeat (bassin versant $143 \mathrm{~km}^{2}$ ). 


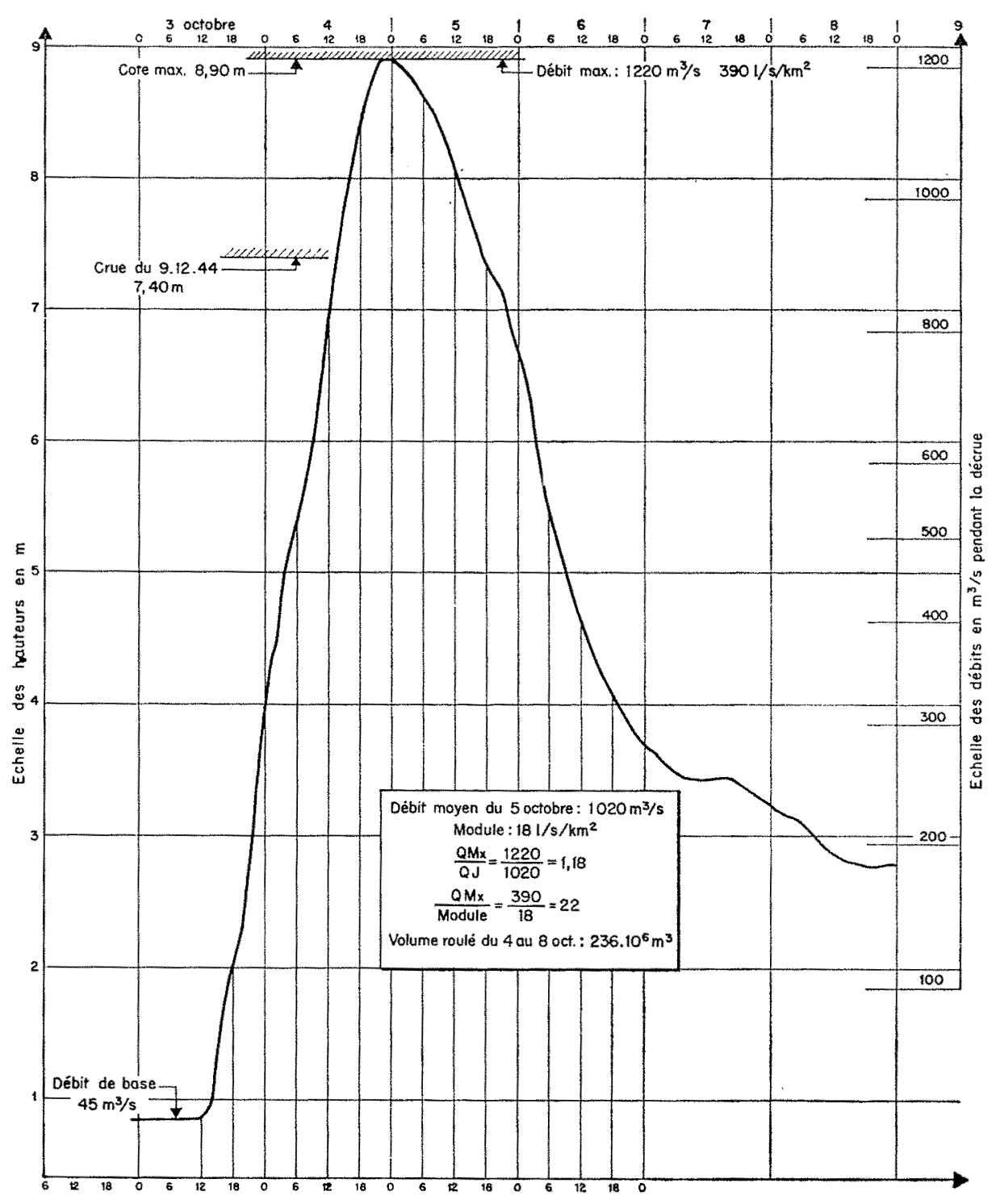

FIG. 4

Hydrogrammes de la Vézère à Montignac (bassin versant $3126 \mathbf{k m}^{2}$ ).

\subsection{Les amplitudes de hauteur}

Le record appartient sans conteste à Montignac, avec plus de $8,00 \mathrm{~m}$ d'amplitude, et où le deuxième étage des maisons de la place d'Armes fut atteint.

A Tulle, la Corrèze noya le quai de Rigny sur une hauteur de $2,40 \mathrm{~m}$. Tous les ponts étaient en charge et l'eau traversait leurs parapets.

A Brive, où l'amplitude fut de même ordre qu'à Tulle, la Corrèze pouvait occuper un très vaste champ d'inondation et les ponts ne furent du moins pas submergés.

A Moulin du Bos, la Vimbelle mit en charge le pont et dut écouler en dehors de lui le tiers de son débit $\left(60 \mathrm{~m}^{3} / \mathrm{s}\right)$, emportant sur cinquante mètres le chemin d'accès au pont.

\subsection{Les temps et vitesses de montée}

Pour les bassins versants inférieurs à $1000 \mathrm{~km}^{2}$ le temps de montée fut cómpris entre 18 et 24 heures.

Les vitesses de montée dépendent évidemment de la section; elles varient de 13 à $68 \mathrm{~cm} /$ heure. Notons que nous sommes très loin des vitesses de montée enregistrées lors des crues de l'Ardèche d'octobre 1958, où, à Sauze-Saint-Martin $\left(2.240 \mathrm{~km}^{2}\right)$ la vitesse de montée fut de $130 \mathrm{~cm} / \mathrm{h}$. 


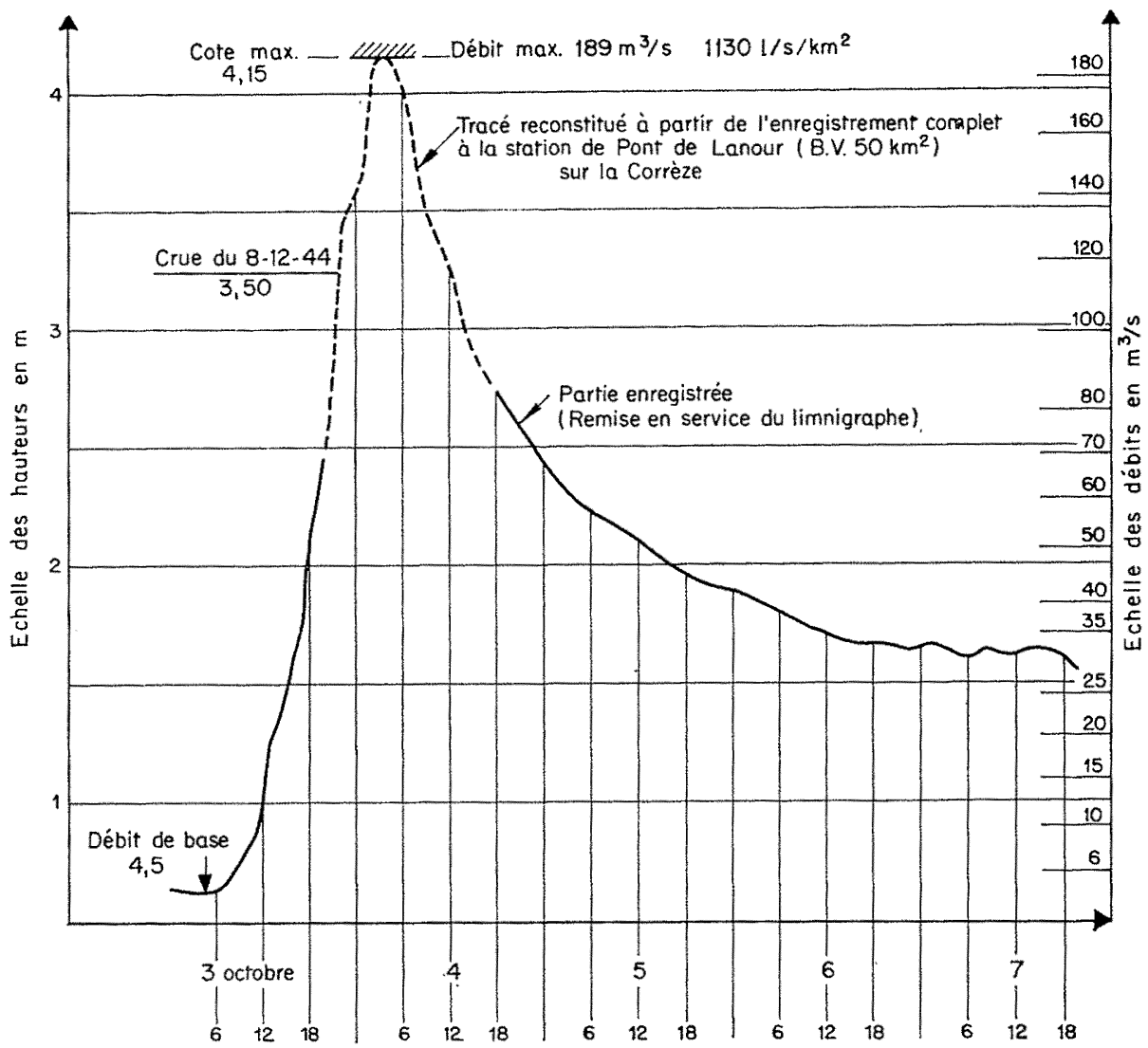

Frg. 5

Hydrogramme de la Corrèze à Corrèze (bassin versant à la station : $167 \mathrm{~km}^{2}$ ).

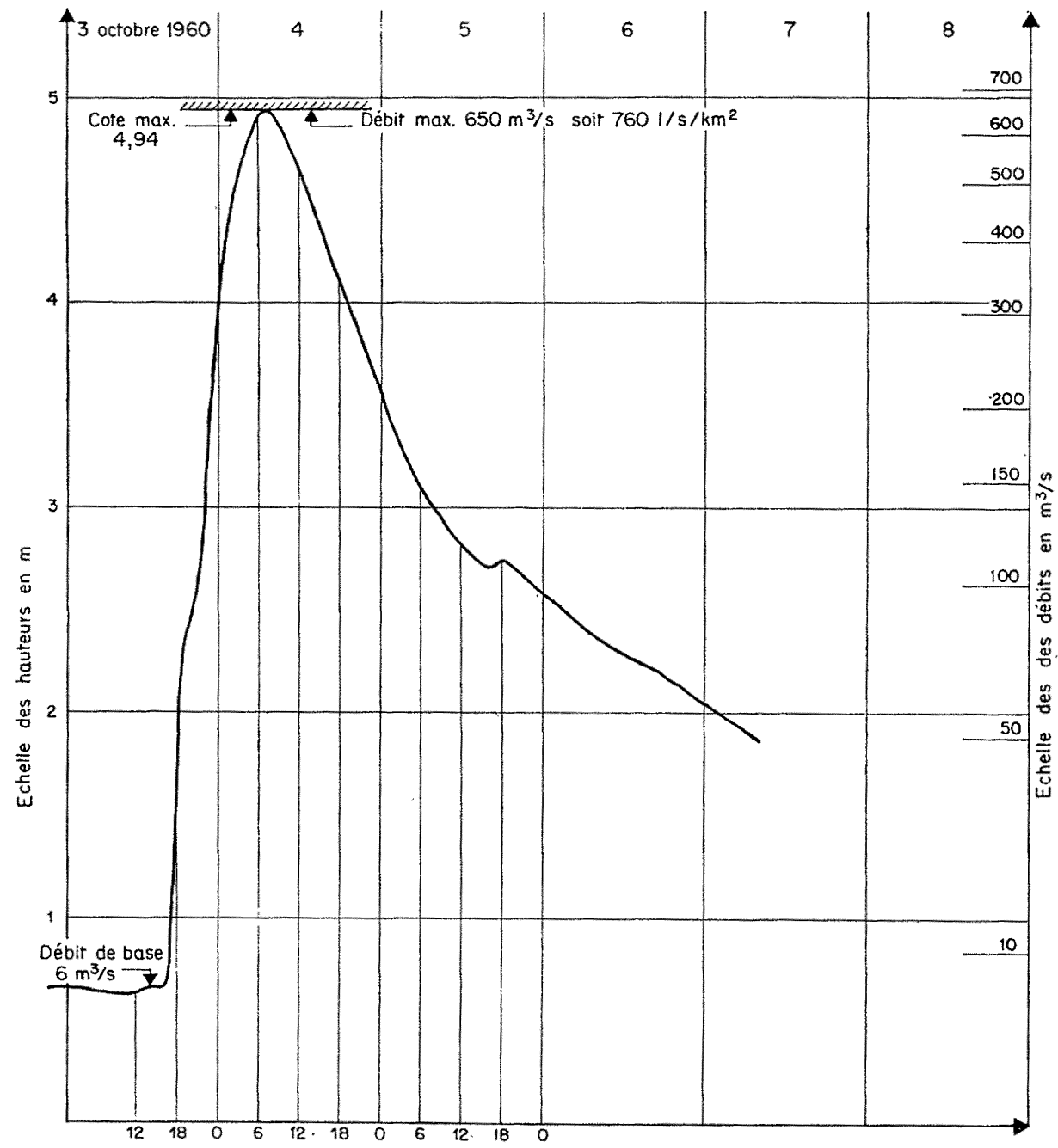

FIG. 6

Hydrogramme de la Tardes à Evaux (bassin versant $860 \mathrm{~km}^{2}$ ).

Enregistrement du limnigraphe complété par les lectures de l'observateur. 


\section{6. - LES DÉBITS SPÉCIFIQUES MAXIMAUX (voir fig. 2, colonnes 5 et 6)}

\subsection{Les valeurs absolues}

Les débits spécifiques maximaux indiqués dans le tableau ci-annexé peuvent paraître faibles à côté de ceux des grandes crues des rivières cévenoles; par exemple, le Chassezac à Thinettes $\left(445 \mathrm{~km}^{2}\right)$ avait charrié $4500 \mathrm{l} / \mathrm{s.} \mathrm{\textrm {km } ^ { 2 }}$ le 4 octobre 1958 , le maximum de la Corrèze à Tulle $\left(370 \mathrm{~km}^{2}\right)$ n'atteint que $1200 \mathrm{l} / \mathrm{s} . \mathrm{km}^{2}$ en octobre 1960.

Mais le plateau de Millevaches n'est pas les Cévennes et il suffit d'avoir vu l'importance des dégâts engendrés par cette crue à Tulle, Brive et Aubusson, pour ne citer que les plus spectaculaires, pour penser à une catastrophe de très rare fréquence, qu'aucun habitant ne se souvient d'avoir vu. Les différentes études statistiques faites pour situer la fréquence de cette crue concordent pour affirmer que celle-ci dé- passe certainement le siècle. Au cours d'une enquête menée spécialement à Montignac, où nous avons consulté soixante-trois années de relevés de hauteurs ainsi que les dessins de construction du pont actuel de Montignac, il fut même impossible de trouver trace, en remontant jusqu'à 1728 , d'une crue de cette importance.

Les maximums affaissés de la Vézère à Uzerche et au Saillant s'expliquent par l'écrêtement assez sensible produit par les retenues des barrages de Montceaux et Treignac qui se trouvaient fortuitement en état d'encaisser des volumes non négligeables. Il en fut de même pour la Vienne au Palais, comme nous l'avons déjà indiqué.

Mis à part ces écrêtements, la répartition des maximums spécifiques est assez homogène, comme le montre le graphique en coordonnées logarithmiques ci-joint (voir fig. 7) : les points

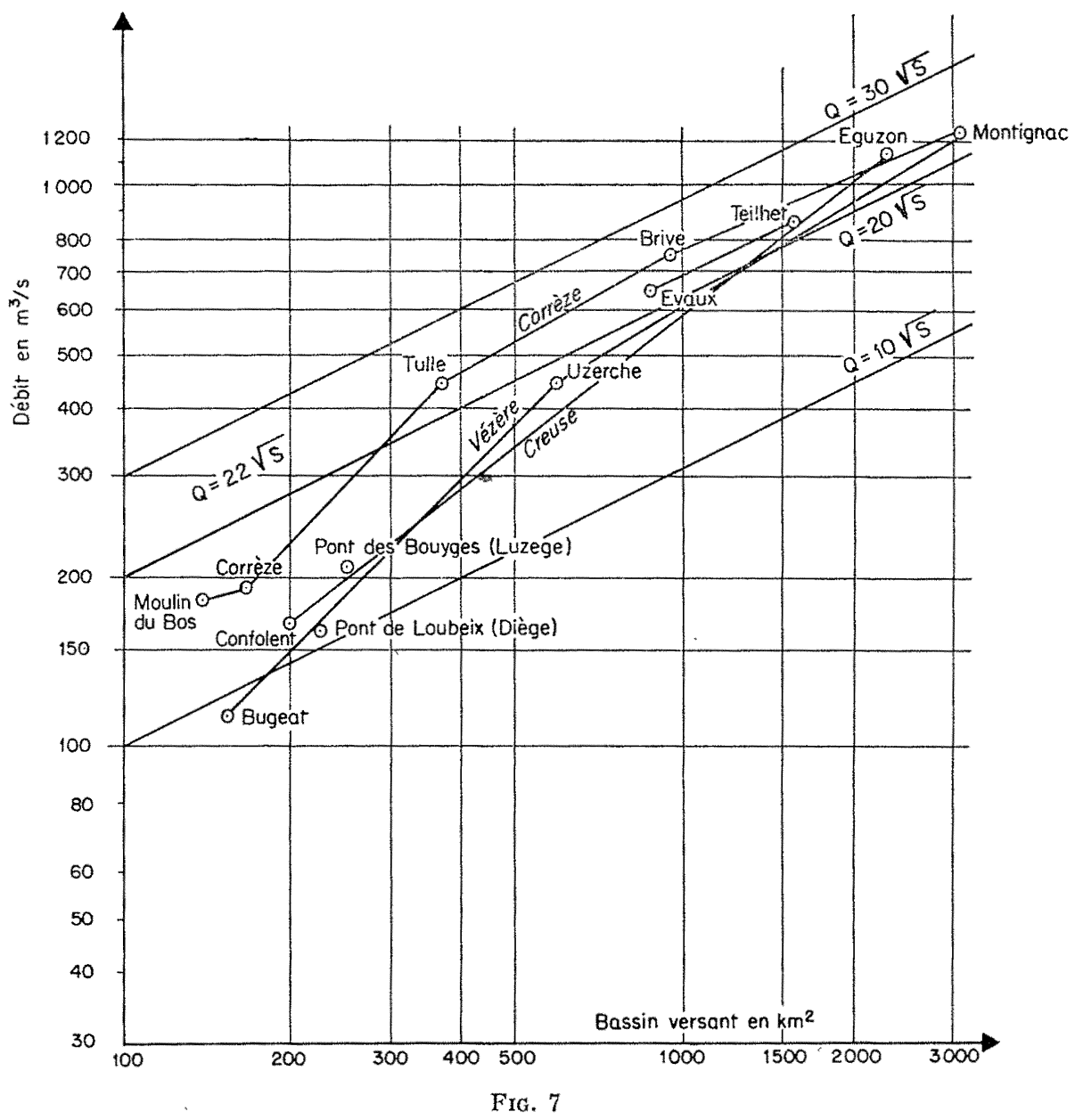

Débits maxịmaux instantanés en fonction des superficies des bassins versants (coordonnées logarithmiques). 
se groupent assez bien autour de la droite $\mathrm{Q}=22 \sqrt{\mathrm{S}}$ lorsque le bassin versant est supérieur à $350 \mathrm{~km}^{2}$; pour les bassins versants plus petits, le groupement se fait nettement au-dessous de cette droite.

\subsection{Les pointes plus ou moins aiguës de la crue}

Le terme $\mathbf{Q}_{m x} / \mathbf{Q}_{J}$, $\mathbf{Q}_{\mathrm{J}}$ désignant le débit moyen $\mathrm{du}$ jour où s'est produit le maximum, peut servir d'image de la pointe plus ou moins aiguë de la crue.

Si on ne retient que les stations où la crue n'a pas été écrêtée par les retenues à l'amont et où le bassin versant est inférieur à $1000 \mathrm{~km}^{2}$, on trouve que ce rapport varie de 1,23 à 2,20 (cf. colonne 8 , fig. 2 ).

La faible valeur de 1,28 à Bugeat est dûe à la faible pente du bassin. Le maximum en cette station, contrôlé par un enregistrement intégral, a duré cinq heures. Il faut y voir aussi l'effet de freinage de «l'éponge» du plateau de Millevaches.

Le chiffre élevé de la Vimbelle, 1,77, comparé à celui de la Corrèze et à plus forte raison de Bugeat, semble indiquer au contraire un ruissellement plus rapide sur les versants abrupts de son bassin. Quant au chiffre de 2,20 de la Diège à Pont de Loubeix, M. d'Harcourt l'explique par la forme du réseau hydrographique qui favorise la concentration du débit et par une forme particulière du hyétogramme moyen.

\subsection{Le caractère exceptionnel représenté par le rapport $\mathrm{Q}_{\mathrm{mx}} /$ Module}

Nous avons mis en évidence ce rapport (cf. colonne 10, fig. 2), car nous pensons qu'il situe bien le degré de relative singularité du maximum par rapport à l'alimentation moyenne de la rivière sur laquelle il s'est produit.

C'est ainsi que ressort d'une façon nette le caractère beaucoup plus exceptionnel de la crue sur la Tardes que sur les autres rivières considé- rées. Alors que ce rapport est compris entre 24 et 52 ailleurs, il est de 68 sur la Tardes à Evaux et de 58 sur le Cher à Teillet à l'aval.

Les maximums spécifiques ne suivent d'ailleurs pas du tout les modules, comme le montre une comparaison des colonnes 6 et 9 du tableau. Ceci confirme que le phénomène météorologique et pluviométrique qui a engendré la crue n'était pas de même nature que ceux qui provoquent les hautes eaux et les petites crues contribuant d'ordinaire à étoffer les modules.

\subsection{Le cas particulier de la Corrèze en amont de Tulle}

A Corrèze, le débit spécifique maximal de la Corrèze a atteint $1130 \mathrm{l} / \mathrm{s} . \mathrm{km}^{2}$, pour un bassin versant de $167 \mathrm{~km}^{2}$. Or à l'aval, à Tulle, où le bassin versant est de $370 \mathrm{~km}^{2}$ et a une altitude moyenne plus faible, le débit spécifique s'est élevé à $1220 \mathrm{I} / \mathrm{s} . \mathrm{km}^{2}$. Cette anomalie entraînait un maximum de $1300 \mathrm{l} / \mathrm{s} . \mathrm{km}^{2}$ pour le bassin intermédiaire, constitué pour les $2 / 3$ par la Vimbelle et pour $1 / 3$ par la Corrèze.

Il n'a pas été possible de déceler dans l'intensité des précipitations, la cause de cette anomalie : les bassins compris entre Corrèze et Tulle, ne paraissent pas avoir reçu des précipitations supérieures à celles reçues par le bassin en amont de Corrèze.

M. Valet, hydrogéologue, a bien voulu se pencher sur ce problème en examinant la constitution géologique du bassin de la Vimbelle. II estime que c'est surtout à la partie inférieure du bassin de cette rivière, en aval de la faille d'Argentat, qu'il faut attribuer un ruissellement plus intense, qui tient alors à deux causes :

$1^{\circ}$ Pour une faible part, la présence, dans la géologie de cette partie du bassin, de nombreux affleurements d'amphibolites imperméables, surtout au sud de Tintignac;

$2^{\circ}$ Pour la plus grande part, la pente de la Vimbelle elle-même, plus forte que celle des rivières voisines et surtout la pente de ses versants qui favorisent un ruissellement fort.

\section{7. - LES LAMES D'EAU ECCOULEES ET PRÉCIPITÉES}

\subsection{Lame écoulée et précipitée en soixante- douze heures}

Nous avons étudié un coefficient de réponse des bassins à la crue sous la forme du rapport K $72=\mathrm{L}_{e} / \mathrm{L}_{p}$ (cf. colonne $13 \mathrm{du}$ tableau de la figure 2), c'est-à-dire le rapport entre la lame écoulée correspondant au débit moyen des soixante-douze heures qui suivent le démarrage de la crue et la lame d'eau précipitée les 3,4 et 5 octobre.

Nous constatons que pour l'ensemble du bassin de la Corrèze, ce rapport ne varie que de 0,52 à 0,55 .

Ce résultat signifie simplement que soixantedouze heures après le début de la crue, la rivière 
a écoulé environ $50 \%$ de la lame précipitée qu'elle a reçue pendant les trois jours du 3 au 5 octobre.

\subsection{Bilan hydrologique sommaire de la crue à Bugeat}

L'hydrogramme de la crue à Bugeat est simple (cf. fig. 3). La courbe de décrue jusqu'au 6 vers midi peut être assez facilement prolongée pour éliminer la fraction du débit due aux pluies qui ont commencé le 7. En prolongeant la courbe de décrue jusqu'à ce qu'on retrouve le débit de base, on détermine la durée à prendre en compte pour le calcul du volume total dû aux seules précipitations des 3, 4 et 5. Le temps écoulé entre le début de la crue et le moment où la rivière retrouve son débit de base est ainsi approximativement de 8 jours $1 / 2$ soit 204 heures.

I.e débit moyen écoulé entre ces deux moments étant de $30,4 \mathrm{~m}^{3} / \mathrm{s}$, dont il faut retrancher le débit de base $2,03 \mathrm{~m}^{3} / \mathrm{s}$, et le bassin versant étant de $143 \mathrm{~km}^{2}$, la lame d'eau correspondant au volume de pluie écoulé devient ainsi :

$$
\mathrm{L}_{e}=\frac{3600 \times 204 \times(30,4-2,0)}{143 \times 10^{6}}=0,145 \mathrm{~m}
$$

d'où un coefficient d'écoulement

$$
\mathrm{K}_{e}=\frac{\mathrm{L}_{e}}{\mathrm{~L}_{p}}=\frac{145}{200}=0,72
$$

Sous réserve de l'exactitude de l'extrapolation de la courbe de décrue, ce rapport a un sens évidemment plus précis que le K 72 du chapitre précédent, à savoir que $28 \%$ environ des précipitations des 3,4 et 5 octobre sont passées en infiltration ou évapotranspiration.

\subsection{Recoupement des volumes roulés}

Nous avons pu contrôler les hydrogrammes de débits en plusieurs stations, en étudiant les volumes roulés par la crue d'une station à une autre et en utilisant les formules empiriques de recoupements résultant des observations faites par notre Service. Nous signalerons seulement que le volume roulé par la Vézère à Montignac du 4 au 8 atteignit 236 millions de mètres cubes, soit $13 \%$ du volume roulé en année moyenne.

\section{8. - COMPARAISON AVEC LES GRANDES CRUES CONNUES ANTERIEUREMENT}

Dans les bassins qui nous intéressent, trois crues relativement récentes s'imposent pour cette comparaison : celle du 8-12-1944 qui a intéressé l'ensemble du versant ouest du Massif Central et du Sancy, celle du 18-9-1921 qui n'a affecté qu'un bassin versant d'une centaine de kilomètres carrés d'un affluent de la Corrèze, enfin celle du 4-5-1940 qui a touché spécialement le bassin de la Tardes et du Cher.

\subsection{La crue du 8 décembre 1944}

Cette crue intéressa un bassin versant environ dix fois plus grand que les $5000 \mathrm{~km}^{2}$ affectés par la crue d'octobre 1960. Par contre, sur ces $5000 \mathrm{~km}^{2}$, la crue de 1944 , tout en étant la plus forte connue depuis 1918, ne dépassa jamais $600 \mathrm{l} / \mathrm{s} . \mathrm{km}^{2}$, soit la moitié des débits de pointe enregistrés en haute-Corrèze en octobre 1960. Les différences de hauteurs atteintes sont d'ailleurs significatives (cf. colonne 15 de la figure 2).

Le phénomène' météorologique qui engendra la crue de décembre 1944 était évidemment, de par sa date même, de nature toute différente de celui d'octobre 1960; la fonte d'un stock de neige important, provoqué par le classique redoux, et des précipitations abondantes et prolongées sont à l'origine de la crue de décembre 1944.

\subsection{La crue du 18 septembre 1921 à Brive}

Dans la nuit du 17 au 18 septembre 1921, la Corrèze subit une crue brutale due à un orage d'une particulière violence qui s'était abaltu sur tous les affluents rive gauche entre Tulle et Brive, soit un bassin d'environ $450 \mathrm{~km}^{2}$. Le débit maximal fut estimé à $470 \mathrm{~m}^{3} / \mathrm{s}$ à Brive; une étude basée sur une relation $Q=f(h)$ nous permet aujourd'hui de confirmer ce chiffre.

C'est un bassin d'une centaine de kilomètres carrés, celui de la Roanne, qui reçut le centre de la précipitation, le reste du bassin versant n'ayant connu que la queue de l'orage. De sorte que sans attribuer à ces seuls $100 \mathrm{~km}^{2}$ les $470 \mathrm{~m}^{3} / \mathrm{s}$ enregistrés à Brive, il est très logique 
de lui en attribuer les deux-tiers, soit un débit spécifique de $3 \mathrm{~m}^{3} / \mathrm{s} . \mathrm{km}^{2}$.

\subsection{La crue du 4 mai 1940 à Teillet}

Cette crue, inférieure par son débit de pointe et par son volume à celle d'octobre 1960 , fut plus homogène : c'est l'ensemble du bassin qui était en crue, alors qu'en 1960, le haut-Cher n'a connu qu'une petite crue, pas même annuelle.
Remarquons d'ailleurs qu'en 1960, si l'axe longitudinal du phénomène météorologique avait été déplacé seulement d'une vingtaine de kilomètres vers le sud-est, le haut-Cher en amont de la Tardes aurait donné un débit spécifique du même ordre que celle-ci et Teillet aurait connu un débit de pointe de peut-être $1300 \mathrm{~m}^{3} / \mathrm{s}$. Une crue de 1200 à $1300 \mathrm{~m}^{3} / \mathrm{s}$ à Montluçon n'est donc pas impensable.

\section{9. - QUELQUES LEÇONS PRATIQUES A TIRER POUR L'EXPLOITATION DES STATIONS DE JAUGEAGE}

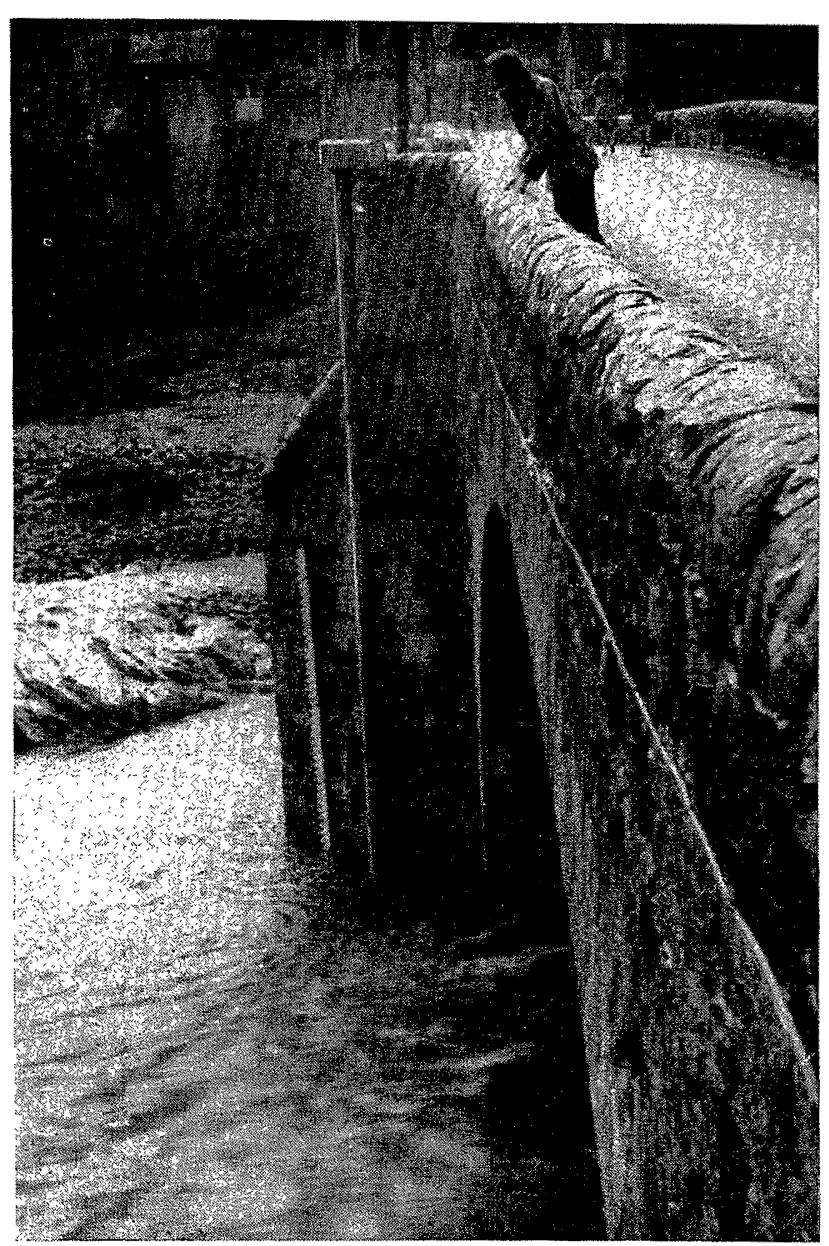

Limnigraphe au pont de Labeaume sur l'Ardèclie.

\subsection{Les limnigraphes}

L'expérience des appareils emportés ou submergés nous invite à chercher à utiliser de plus en plus les ouvrages existants pratiquement insubmersibles comme les ponts. A ce moment, se posent des questions d'esthétique et d'encombrement, problèmes auxquels nous avons cherché à donner des solutions pratiques. Les photos cicontre montrent quelques exemples de réalisations faites dans cet esprit.

\subsection{Les sections auxiliaires}

L'expérience montre que l'évaluation des débits de crue peut rarement être faite en une seule section. Il faut toujours se recouper sur une deuxième section. Cela suppose qu'on a choisi celle présentant les conditions les plus favorables (aux débordements les plus réduits) et qu'on y a installé une échelle auxiliaire permettant le tracé facile de la courbe des sections mouillées $\mathrm{S}=f(h)$, ce qui, bien entendu, a été fait jusqu'aux plus hautes cotes au droit de l'échelle principale.

La mesure des pentes superficielles se trouvera toujours grandement facilitée par l'installation d'échelles auxiliaires aux zéros rattachés au N.G.F. L'une de ces sections pourra être celle retenue pour l'évaluation des crues, l'autre étant choisie de manière que l'échelle principale se trouve encadrée par les deux sections. 


\section{0. - CONCLUSION}

Les deux dates du 30 septembre -4 octobre 1958 sur l'Ardèche et du 4 octobre 1960 à l'ouest du plateau de Millevaches, resteront dans la mémoire et les archives des hydrauliciens français.

La crue que nous venons d'étudier est non seulement la plus forte de celles ayant intéressé un bassin versant de plus de $1000 \mathrm{~km}^{2}$ du versant ouest du Massif Central, mais aussi la première de cette région qui ait pu être sérieusement étudiée dans ses précipitations comme dans son écoulement.

Nous n'avons certes pas épuisé les enseignements de cette crue. Bien des points restent à éclaircir, notamment la question des coefficients de ruissellement et des bilans d'écoulement, l'influence de la pente des versants dans la genèse des débits de pointe, l'influence retardatrice de "l'éponge » du plateau de Millevaches.

De l'analyse des débits qui a été faite, il ressort d'une manière certaine, qu'avec ses pointes ayant atteint et parfois dépassé $1000 \mathrm{l} / \mathrm{s} . \mathrm{km}^{2}$, il s'agissait d'une crue plus que centenaire. Ce fait est d'ailleurs confirmé par les études statistiques auxquelles nous avons procédé par ailleurs en quelques stations.

Sans que les débits de pointe y atteignent les chiffres spectaculaires des rivières cévenoles, il faut redouter les crues d'automine sur le versant ouest du Massif Central. Jusqu'à ce jour, en effet, on avait tendance à estimer que les crues

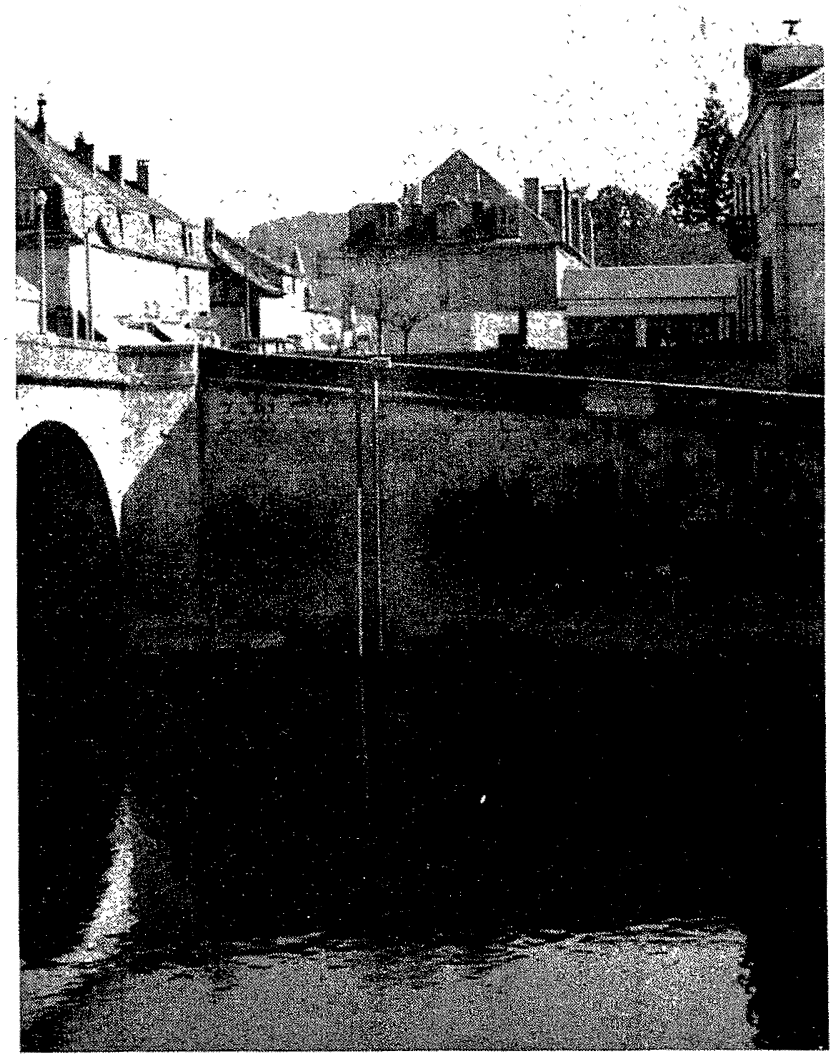

Limnigraphe à Montignac-sur-Vézère. Vue d'ensemble.
Type d'installation d'appareil

- Capacité d'enregistrement limitée seulement par la submersion.

- Diamètre de la gaine : $20 \mathrm{~cm}$.

- Facilité d'installation.

- Discrétion sur l'ouvrage auquel il est fixé.

Limnigraphe à Montignac-sur-Vézère. L'appareil à portée de l'observateur.

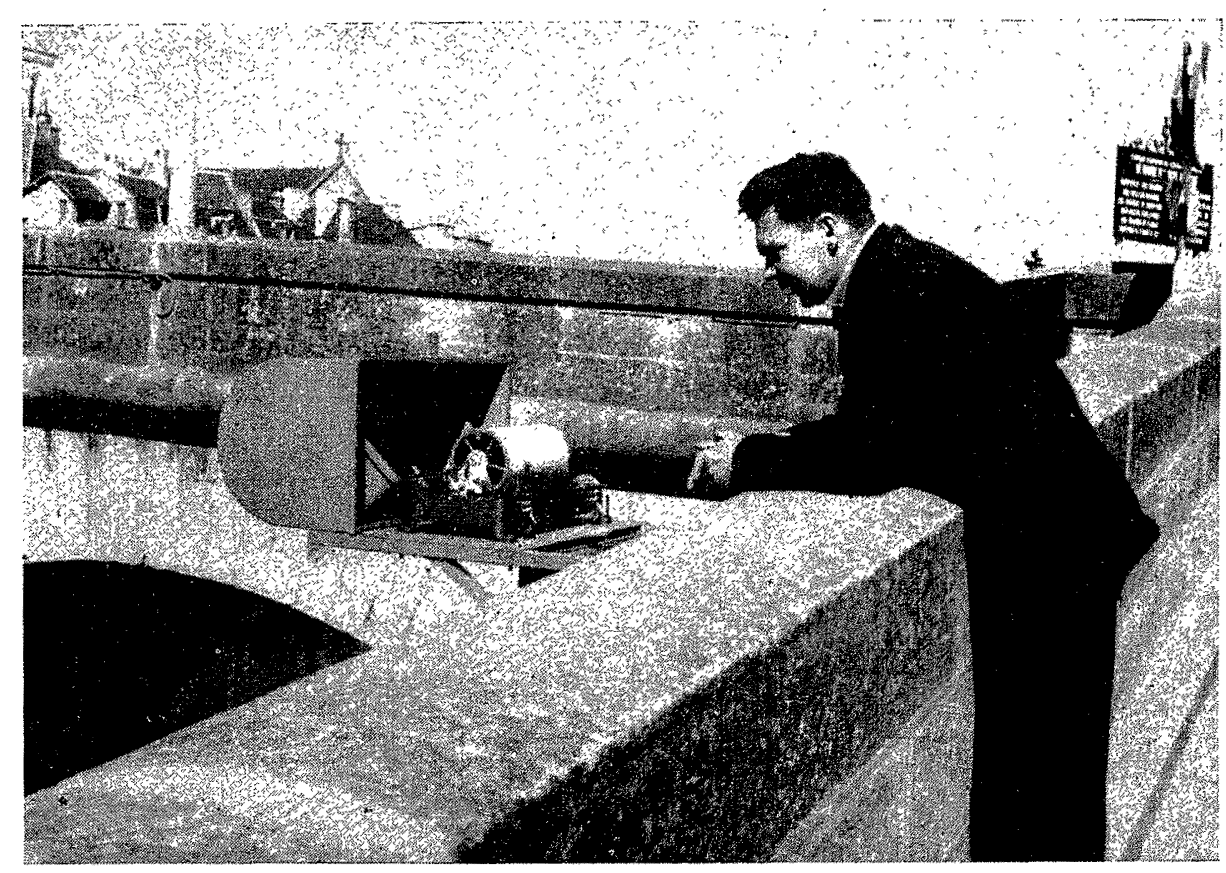


les plus importantes sur ce versant étaient dues à des pluies tombant sur un manteau neigeux, avec radoucissement général de la température (crue type décembre 1944). Ce schéma est à réviser, d'autant plus qu'à l'occasion de cette étude, nous avons rappelé la trombe orageuse du 18 septembre 1921 qui a provoqué sur la Corrèze en amont de Brive, à l'échelle de la centaine de $\mathrm{km}^{2}$ de bassin versant, un débit spécifique de pointe de deux à trois fois supérieur à ceux d'octobre 1960.
P.S. - Nous sommes très reconnaissants à MM. Jes Ingénieurs des Services des Ponts et Chaussées de Tulle, Brive et Evaux et des Services d'Electricité de France pour leur collaboration aux opérations sur le terrain nécessitées par cette étude. Nous tenons également à remercier ici, MM. Jacquet du Service des Etudes et Recherches Hydrauliques à E.D.F., M. Valet, Hydrogéologue, M. Lacroix, Ingénieur des Ponts et Chaussées à Périgueux, et M. Pardé, des utiles informations échangées à propos de cette étude.

\section{I S C U S S I O N}

Président: M. DE RouviLLE

M. le Président donne la parole à M. BARRIène qui remercie $M$. JacQuer de la façon dont il a résumé son rapport. Comme il a été beaucoup question d'une comparaison avec la crue du 30-9-1958 dans l'Ardèche, il donne, à titre de comparaison, quelques chiffres de débits maximaux pour des bassins versants du même ordre :

- le 4 octobre 1960 :

pour la Corrèze à Tulle : $1200 \mathrm{l} / \mathrm{s} . \mathrm{km}^{2}$ pour un bassin versant de $370 \mathrm{~km}^{2}$;

- le 30 septembre 1958 :

pour le Chassezac: $4500 \mathrm{l} / \mathrm{s.} \mathrm{km}^{2}$ pour un bassin versant de $445 \mathrm{~km}^{2}$.

Si l'on prend des bassins plus importants - comme la Vézère à Montignac qui totalise le bassin de la Vézère à l'aval de la Corrèze, - pour environ $3000 \mathrm{~km}^{2}$ de bassin versant, on obtient le maximum modeste de $390 \mathrm{l} / \mathrm{s} . \mathrm{km}^{2}$. Mais, le 30 septembre 1958, l'Ardèche à Saint-Martin d'Ardèche, pour $2200 \mathrm{~km}^{2}$, atteignait $2100 \mathrm{l} / \mathrm{s} . \mathrm{km}^{2}$, donc $2 \mathrm{~m}^{3} / \mathrm{s} . \mathrm{km}^{2}$.

D'autre part, la Vimbelle a bien été un élément perturbateur. Au lieu de contribuer à aplatir, de l'amont à l'aval, le' débit maximal de la crue, elle l'a renforcé, puisque la Corrèze à Corrèze atieignait $1100 \mathrm{l} / \mathrm{s} . \mathrm{km}^{2}$ et à Tulle $1200 \mathrm{l} / \mathrm{s} . \mathrm{km}^{2}$ pour $370 \mathrm{~km}^{2}$. Cela est attribué, à la suite d'une étude de M. VALET, à la constitution géologique du bassin de la Vimbelle, comportant une proportion de terrains imperméables plus importante que dans le bassin de la Corrèze à Corrèze et surtout une pente des versants beaucoup plus accen‘uée.

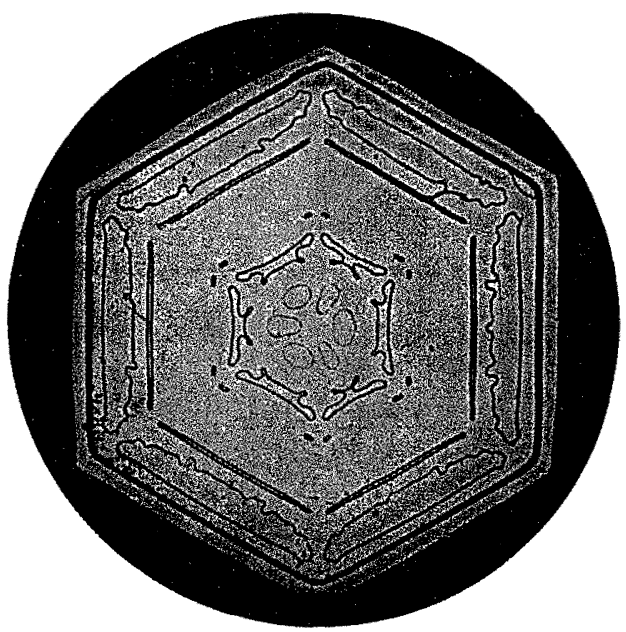

